\title{
Особенности распространения стоячих электромагнитных и электронных волн в металлическом проводнике с электрическим переменным током проводимости
}

\author{
М. И. Баранов \\ Научно-исследовательский и проектно-конструкторский институт «Молния» \\ Национального технического университета «Харьковский политехнический институт», \\ 2. Харьков, 61013, Украина e-mail: baranovmi@kpi.kharkov.ua \\ Поступила в редакцию 27.04.2021 \\ После доработки 31.05.2021 \\ Принята к публикации 02.06.2021
}

\begin{abstract}
Приведены результаты приближенных расчетов по установлению основных особенностей распространения стоячих поперечных электромагнитных волн (ЭМВ) и стоячих продольных электронных волн де Бройля в однородном немассивном немагнитном металлическом проводнике конечных размеров (радиусом $r_{0}$ и длиной $l_{0}>>r_{0}$ ) с переменным аксиальным током проводимости $i_{0}(t)$ различных амплитудно-временных параметров. Получены соотношения для расчетной оценки усредненных скоростей распространения для стоячих поперечных ЭМВ и стоячих продольных электронных волн де Бройля в металле (сплаве) указанного проводника. Показано, что возникающие в металлическом проводнике конечных размеров квантованные стоячие поперечные ЭМВ существенно отличаются от обычных поперечных ЭМВ, распространяющихся в проводящих средах неограниченных размеров. Важной особенностью стоячих поперечных ЭМВ в рассматриваемом проводнике является то, что их напряженность аксиального электрического поля опережает по фазе их напряженность азимутального магнитного поля на угол $\pi / 2$. Установлено, что в стоячих поперечных ЭМВ исследуемого проводника энергия электрического поля лишь переходит в энергию их магнитного поля и наоборот. Поэтому стоячие поперечные ЭМВ не переносят по металлу (сплаву) рассматриваемого проводника потоков электромагнитной энергии.
\end{abstract}

Ключевые слова: металлический проводник, электрический переменный ток проводимости, стоячие поперечные электромагнитные волны, стоячие продольные электронные волны де Бройля, скорость распространения волн

УДК 621.3.01: 621.313

https://doi.org/10.52577/eom.2021.57.6.72

\section{СОСТОЯНИЕ И АКТУАЛЬНОСТЬ ЗАДАЧИ}

В [1-5] с учетом квантово-волновой природы электрического тока проводимости $i_{0}(t)$ различных видов (постоянного, переменного и импульсного) и амплитудно-временных параметров (АВП) в металле (сплаве) цилиндрических проводников конечных геометрических размеров (радиусом $r_{0}$ и длиной $l_{0}>>r_{0}$ ) были получены новые электрофизические данные, касающиеся протекания волновых электромагнитных процессов в их однородном немагнитном электропроводящем материале. При этом на основе существования в проводящей структуре указанных проводников квантованных продольных электронных волн (длиной $\lambda_{n z} \leq 2 l_{0}$ ) или полуволн (длиной $\lambda_{n z} / 2 \leq l_{0}$ ) де Бройля [6], обусловленных дрейфом в ней коллективизированных свободных электронов, и их пространственно-временных распределений в этой структуре было впервые в области электротехники (электрофизики) теоретически и экспериментально (в лабораторных условиях при комнатной температуре $\left(20^{\circ} \mathrm{C}\right.$ [7]) и плотности импульсного тока $i_{0}(t)$ в круглом стальном проводе около 0,37 кА/мм ${ }^{2}$ [3-5]) установлено проявление в этих проводниках нового квантового электрофизического эффекта, связанного с образованием в их металле (сплаве) продольных волновых электронных пакетов (ВЭП) и возникновением в проводниках из-за влияния образующихся ВЭП продольной периодической локализации дрейфующих электронов. Данная локализация основных носителей электричества в металлических проводниках приводит к появлению в их материале «горячих» длиной $\Delta z_{n h}$ и «холодных» длиной $\Delta z_{n c}$ квантованных продольных участков (см. рисунок) с резко отличающимися (максимум в 3,5 раза [3-5]) температурами джоулева нагрева. Причем шаг такой периодически размещенной вдоль их длины $l_{0}$ тепловой макроструктуры составляет величину, равную $\lambda_{n z} / 2=\left(\Delta z_{n h}+\Delta z_{n c}\right)$ [2-5], где $n=1,2,3, \ldots, n_{m}-$ целое квантовое число; $n_{m}=2 n_{0}^{2}-$ максимальное значение квантового числа $n ; n_{0}$ - главное квантовое число, равное 
числу электронных оболочек в атомах металла проводника или номеру периода в Периодической системе элементов Менделеева, которому этот металл принадлежит (например, для медного, цинкового и стального проводников $n_{0}=4$, а $n_{m}=32$; для алюминиевого проводника $n_{0}=3$, a $\left.n_{m}=18[6]\right)$.

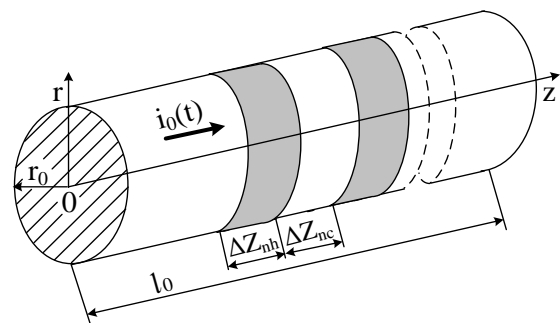

Общий схематический вид круглого цилиндрического проводникарадиусом $r_{0}$ и длиной $l_{0}>>r_{0}$ с аксиальным током проводимости $i_{0}(t)$, где $\Delta z_{n h}, \Delta z_{n c}-$ соответственно длины »горячего» и «холодного» продольных участков проводника [4].

Согласно [2-5], в металле (сплаве) цилиндрического проводника с переменным аксиальным током проводимости $i_{0}(t)$ различных АВП возникают стоячие поперечные электромагнитные волны (ЭМВ) $[8,9]$ с квантованными продольными компонентами напряженности $E_{n z}$ электрического и азимутальными компонентами напряженности $H_{n v}$ магнитного полей, расположенными в плоскостях, параллельных продольной оси $O Z$ проводника (см. рисунок). Эти стоячие поперечные ЭМВ, вектор Пойнтинга которых направлен по радиусу $r_{0}$ внутрь проводящего материала проводника, не способны к передаче в металле (сплаве) проводников и вдоль воздушных (кабельных) линий с данными проводниками и соответственно на расстояние потоков электромагнитной энергии от ее источника к потребителю [6, 9]. Порядок квантования (дискредитации) стоячих поперечных ЭМВ в проводниках определяется указанным порядком квантования в них продольных электронных волн де Бройля длиной $\lambda_{n z}$, вызывающих появление в их металле (сплаве) продольных волн тока $i_{0}(t)$ и напряжения $u_{0}(t)$ и соответствующих стоячих ЭМВ. Первой принципиальной особенностью проявления квантованных волновых электромагнитных процессов в металлических проводниках с током проводимости $i_{0}(t)$ является то, что на их длине $l_{0}$ всегда укладывается только целое число продольных электронных полуволн де Бройля длиной $\lambda_{n z} / 2$, удовлетворяющей квантовомеханическому соотношению вида [1-5]: $\lambda_{n z} / 2=l_{0} / n$. Второй важной особенностью рассматриваемых волновых электромагнитных процессов в указанных проводниках является то, что они имеют место даже для квазистационарного режима протекания тока проводимости $i_{0}(t)$ в электрической цепи с ними, для которого характерно выполнение следующего условия [4, 10]: $\lambda_{0}>2 l_{0}$, где $\lambda_{0}-$ длина бегущей поперечной ЭМВ в газовом (твердом) диэлектрике, окружающем проводники. При изучении подобных волновых электромагнитных процессов в данных проводниках и определении их влияния на электрофизические свойства токонесущих частей кабельно-проводниковой продукции (например, на показатели ослабления их материалом проникающего в них переменного электрического поля и электротехнические возможности этих частей проводников при передаче на расстояние электромагнитной энергии) одной из научных задач является та, которая связана с изучением особенностей распространения квантованных стоячих поперечных ЭМВ длиной $\lambda_{n r}$ и стоячих продольных электронных волн де Бройля длиной $\lambda_{n z}$ в их металле. При этом прежде всего требуется уметь определять расчетным путем скорости распространения указанных стоячих волн (полуволн) в проводящем материале исследуемых проводников с аксиальным током проводимости $i_{0}(t)$ тех или иных АВП. В настоящее время подобные расчетные соотношения для указанных скоростей стоячих поперечных ЭМВ и стоячих электронных волн де Бройля в металлических проводниках с током проводимости $i_{0}(t)$ различных АВП, широко применяемых в области электротехники, электроэнергетики и высоковольтной импульсной техники, отсутствуют.

Цель статьи - приближенный расчет усредненных скоростей распространения квантованных стоячих поперечных ЭМВ и квантованных стоячих продольных электронных волн де Бройля в металлических проводниках с переменным аксиальным током проводимости $i_{0}(t)$ различных АВП.

\section{ПОСТАНОВКА ЗАДАЧИ}

Рассмотрим размещенный в воздушной среде круглый сплошной немассивный цилиндрический однородный проводник радиусом $r_{0}$ и длиной $l_{0}>>r_{0}$ (см. рисунок) с удельной электропроводностью $\gamma_{0}$ его немагнитного металла (сплава) и сплошным поперечным сечением $S_{0}$, по которому в продольном направлении протекает электрический переменный ток проводимости $i_{0}(t)$ произвольных АВП. Принимаем, что АВП аксиального синусоидального тока $i_{0}(t)$ изменяются во времени $t$ с частотой колебаний $f_{0}$ и допускают его практически равномерное распределение по сечению $S_{0}$ проводника. Считаем, что продольное волновое распределение дрейфующих свободных электронов в 
данном проводнике подчиняется одномерным квантованным волновым функциям Шредингера $\psi_{n z}(z, t)[4]$.

Принимаем, что квантованные продольные волновые функции Шредингера $\psi_{n z}(z, t)$, носящие гармонический характер своего пространственно-временного изменения [1-4], определяют продольные изменения электронных волн (полуволн) де Бройля в металле исследуемого проводника. Процесс диффузии стоячих поперечных ЭМВ в однородный металл (сплав) исследуемого проводника рассмотрим для случая, когда кривизной наружной цилиндрической поверхности проводника в рассматриваемых полевых расчетах можно пренебречь и проникающую в его глубину монохроматическую поперечную ЭМВ в первом приближении можно считать плоской. Током смещения в металле (сплаве) проводника по сравнению с током проводимости $i_{0}(t)$ пренебрегаем [11]. C учетом квантово-механического подхода к продольному волновому распределению дрейфующих свободных электронов в кристаллической структуре исследуемого проводника расчетным путем требуется определить в принятом приближении как квантованную скорость $v_{n r}$ распространения стоячих поперечных ЭМВ, так и квантованную скорость $v_{n z}$ распространения стоячих продольных электронных волн де Бройля в однородном немагнитном металле круглого сплошного цилиндрического проводника с током $i_{0}(t)$.

\section{РАСЧЕТНАЯ ОЦЕНКА УСРЕДНЕННОЙ СКОРОСТИ РАСПРОСТРАНЕНИЯ СТОЯЧИХ ПОПЕРЕЧНЫХ ЭМВ В МАТЕРИАЛЕ ПРОВОДНИКА}

Из [1-5] для квантованных значений напряженности $E_{n z}$ продольного электрического и напряженности $H_{n v}$ азимутального магнитного полей в плоской стоячей поперечной ЭМВ на наружной цилиндрической поверхности $\left(r=r_{0}\right)$ рассматриваемого однородного проводника с аксиальным синусоидальным током проводимости $i_{0}(t)$ можно получить следующие аналитические соотношения:

$$
\begin{aligned}
& H_{n v}\left(r_{0}, z, t\right) \approx 0,5 \delta_{0 m} r_{0} \sin \left(k_{n z} z\right) \sin \left(2 \pi f_{0} t\right) ; \\
& E_{n z}\left(r_{0}, z, t\right) \approx \delta_{0 m} \gamma_{0}^{-1} \cos \left(k_{n z} z\right) \cos \left(2 \pi f_{0} t\right),
\end{aligned}
$$

где $k_{n z}=2 \pi / \lambda_{n z}=\pi n / l_{0}-$ квантованное продольное волновое число; $n=1,2,3 \ldots, n_{m}$ - целое квантовое число; $\delta_{0 m} \approx I_{0 m} / S_{0}-$ амплитуда плотности тока в металле (сплаве) проводника с удельной электропроводностью $\gamma_{0} ; I_{0 m}$ - амплитуда тока проводимости $i_{0}(t)$, протекающего в проводнике.

Далее, не теряя общих свойств (параметров) при определении величины скорости $v_{n r}$ распро- странения стоячей поперечной ЭМВ в металле проводника, остановимся на рассмотрении случая, когда в (1) и (2) аргумент $\left(k_{n z} z\right)$ в их пространственных гармонических функциях будет численно равным $\pi / 4$. Тогда квантованные соотношения (1) и (2) для продольной координаты $z=l_{0} /(4 n)$ исследуемого проводника с током проводимости $i_{0}(t)$, соответствующие напряженностям переменного электромагнитного поля (ЭМП) на его наружной цилиндрической поверхности, запишем в следующем упрощенном виде:

$$
\begin{gathered}
H_{v 0}\left(r_{0}, t\right) \approx 0,353 \delta_{0 m} r_{0} \sin \left(2 \pi f_{0} t\right) ; \\
E_{z 0}\left(r_{0}, t\right) \approx 0,707 \delta_{0 m} \gamma_{0}^{-1} \cos \left(2 \pi f_{0} t\right) .
\end{gathered}
$$

Перепишем (4) в виде следующей тригонометрической зависимости:

$$
E_{z 0}\left(r_{0}, t\right) \approx 0,707 \delta_{0 m} \gamma_{0}^{-1} \sin \left(2 \pi f_{0} t+\pi / 2\right) .
$$

Используя известный электротехнический подход при изучении распространения плоской монохроматической поперечной ЭМВ в проводящей среде неограниченных геометрических размеров (проводящем полупространстве) [11], для процесса линейной диффузии стоячей поперечной ЭМВ плоскомеридианного ЭМП в металл (сплав) исследуемого цилиндрического проводника конечных размеров с учетом (3)-(5) можно в случае, когда для его материала продольная координата равна $z=l_{0} /(4 n)$, в приближенном виде записать следующие электродинамические выражения:

$$
\begin{aligned}
& H_{v}(r, t) \approx 0,353 \delta_{0 m} r_{0} e^{-k_{r}\left(r_{0}-r\right)} \times \\
& \times \sin \left[2 \pi f_{0} t-k_{r}\left(r_{0}-r\right)\right] ; \\
& E_{z}(r, t) \approx 0,707 \delta_{0 m} \gamma_{0}^{-1} e^{-k_{r}\left(r_{0}-r\right)} \times \\
& \times \sin \left[2 \pi f_{0} t-k_{r}\left(r_{0}-r\right)+\pi / 2\right],
\end{aligned}
$$

где $k_{r}=\left(\pi f_{0} \mu_{0} \gamma_{0}\right)^{1 / 2}=2 \pi \lambda_{n r}=2 \pi \lambda_{r}-$ радиальное волновое число для материала проводника, испытывающего воздействие проникающей в него ЭМВ; $\mu_{0}=4 \pi \times 10^{-7} \Gamma \mathrm{H} / \mathrm{M}-$ магнитная постоянная [7].

Принципиальным отличием полученных полевых соотношений (6) и (7) от приведенных в [11] является то, что во всех точках металла (сплава) круглого проводника, включая и его наружную поверхность $\left(r_{0}-r=0\right)$, для стоячей поперечной ЭМВ напряженность $E_{z}(r, t)$ продольного электрического поля опережает по фазе напряженность $H_{v}(r, t)$ азимутального магнитного поля на угол, равный $\pi / 2$. Отметим, что для плоской монохроматической поперечной ЭМВ в массивной проводящей среде при отсутствии в ней отраженных ЭМВ этот угол 
численно составляет $\pi / 4$ [11]. Именно это отличие и является основной полевой особенностью, характерной для стоячих поперечных ЭМВ, распространяющихся в металле исследуемого немассивного проводника с аксиальным током проводимости $i_{0}(t)$.

Из (6) и (7) следует, что по мере радиального проникновения в однородный металл (сплав) исследуемого проводника стоячей поперечной ЭМВ вектор Пойнтинга $\vec{P}_{n}$, которой по радиусу $r$ направлен в глубь его материала, ее напряженности $E_{z}(r, t)$ аксиального электрического и напряженности $H_{v}(r, t)$ азимутального магнитного полей убывают также, как и для плоской обычной поперечной ЭМВ по экспоненциальному закону [11]. Причем в исследуемом случае начальная фаза колебаний напряженностей $E_{z}(r, t)$ и $H_{v}(r, t)$, характерных для стоячей поперечной ЭМВ в рассматриваемом немассивном проводнике, аналогична соответствующим напряженностям для плоской обычной поперечной ЭМВ в массивной проводящей среде [11], также изменяется прямо пропорционально пройденному ею текущему расстоянию $\left(r_{0}-r\right)$ от наружной поверхности проводника $\left(r=r_{0}\right)$ до его центра $(r=0)$. Кроме того, по мере проникновения стоячей поперечной ЭМВ плоскомеридианного ЭМП в глубь металла цилиндрического проводника колебания ее напряженностей $E_{z}(r, t)$ и $H_{v}(r, t)$, как и для плоской обычной поперечной ЭМВ [11], запаздывают все более по фазе по отношению к их колебаниям на наружной цилиндрической поверхности проводника $\left(r=r_{0}\right)$ с током проводимости $i_{0}(t)$.

Переносят ли стоячие поперечные ЭМВ с указанными, согласно (6) и (7), напряженностями ЭМП по металлу (сплаву) рассматриваемого проводника с аксиальным током проводимости $i_{0}(t)$ электромагнитную энергию? Ответ на данный вопрос может дать расчет среднего за период колебаний $T_{0}=1 / f_{0}$ тока проводимости $i_{0}(t)$ значения указанного выше вектора Пойнтинга $\vec{P}_{n}$, направленного по текущему радиусу $r$ внутрь исследуемого металлического проводника. Используя (6), (7) и известные положения теории ЭМП $[6,11]$, для среднего за период колебаний $T_{0}=1 / f_{0}$ протекающего по исследуемому проводнику тока проводимости $i_{0}(t)$ значения модуля вектора Пойнтинга $P_{n}$ получаем:

$$
\begin{aligned}
& P_{n} \approx 0,125 \delta_{0 m}^{2} \gamma_{0}^{-1} r_{0} f_{0} e^{-2 k_{r}\left(r_{0}-r\right)} \times \\
& \times \int_{0}^{f_{0}^{-1}} \sin \left[4 \pi f_{0} t-2 k_{r}\left(r_{0}-r\right)\right] d t \approx 0 .
\end{aligned}
$$

Из (8) вытекает важный электротехнический вывод: возникающие в рассматриваемом проводнике стоячие поперечные ЭМВ электромагнитную энергию по его металлу (сплаву) не переносят. Данный вывод соответствует существующим положениям теории стоячих ЭМВ [6, 8]. При этом в стоячих ЭМВ исследуемого проводника энергия продольного электрического поля с напряженностью $E_{z}(r, t)$ переходит в энергию его азимутального магнитного поля с напряженностью $H_{v}(r, t)$ и наоборот (причем этот переход не сопровождается потерями электромагнитной энергии).

Что касается расчетного определения квантованной скорости $V_{n r}$ распространения стоячей поперечной ЭМВ в металлическом проводнике, то она в рассматриваемом случае (для $k_{n z} Z=\pi / 4$ в соотношениях (1) и (2)) вырождается в обычную радиальную скорость $v_{r}$ распространения плоскомеридианной стоячей поперечной ЭМВ обычной длиной $\lambda_{n r}=\lambda_{r}$ в однородном металле исследуемого проводника. Помня, что длина $\lambda_{r}$ стоячей поперечной ЭМВ соответствует пройденному ею в изотропном металле проводника расстоянию $\left(r_{0}-r\right)=\lambda_{r}$, на котором фаза колебаний ее напряженностей $E_{z}(r, t)$ и $H_{v}(r, t)$ изменяется на $2 \pi$, из (6) и (7) вытекает следующее электродинамическое равенство:

$$
k_{r}\left(r_{0}-r\right)=k_{r} \lambda_{r}=2 \pi .
$$

Подставив в (9) из (6) или (7) значение радиального волнового числа $k_{r}=\left(\pi f_{0} \mu_{0} \gamma_{0}\right)^{1 / 2}$, для длины $\lambda_{r}$ плоскомеридианной стоячей поперечной ЭМВ в металле (сплаве) рассматриваемого проводника конечных размеров (радиусом $r_{0}$ и длиной $l_{0}>r_{0}$ ) с аксиальным током проводимости $i_{0}(t)$ получаем:

$$
\lambda_{r}=2 \pi /\left(\pi f_{0} \mu_{0} \gamma_{0}\right)^{1 / 2} .
$$

Укажем, что формула (10) соответствует известному аналогичному расчетному соотношению, приведенному в [11] и характерному для длины плоской монохроматической обычной поперечной ЭМВ, распространяющейся в неограниченной по размерам линейной проводящей среде (проводящем полупространстве), где будут отсутствовать отраженные поперечные ЭМВ и где не будет электрофизических условий для возникновения стоячих поперечных ЭМВ. Это обстоятельство свидетельствует о том, что, несмотря на указанное отличие стоячих поперечных и обычных поперечных ЭМВ в однородном металле (сплаве) проводящей среды, они имеют общую природу своего происхождения и описываются классической теорией электромагнитного поля, разработанной Максвеллом [8]. 
Расчетная оценка по (10) длины $\lambda_{r}$ стоячей поперечной ЭМВ в круглом медном проводнике $\left(\gamma_{0}=5,81 \times 10^{7} \mathrm{Cm} / \mathrm{M}\right.$ [7] $)$ при $f_{0}=50$ кГц показывает, что в этом случае она оказывается примерно равной 1,85 мм. Согласно (6) и (7), на такой глубине $\left(r_{0}-r=\lambda_{r}\right)$ напряженности $E_{z}(r, t)$ и $H_{v}(r, t)$ испытывают свое практически полное затухание, так как в этом электрофизическом случае $\exp \left(-k_{r} \lambda_{r}\right)=\exp (-2 \pi) \approx 0,0018$.

В то же время для длины $\lambda_{r}$ плоскомеридианной стоячей поперечной ЭМВ в приповерхностном слое $\left(r \rightarrow r_{0}\right)$ проводника характерно приближенное соотношение следующего вида: $\lambda_{r} \approx v_{r} / f_{0}$. При проникновении данной ЭМВ в глубь металла (сплава) проводника, согласно (6) и (7), будет происходить заметное уменьшение частоты колебаний $f$ волн напряженностей продольного электрического $E_{z}(r, t)$ и азимутального магнитного $H_{v}(r, t)$ полей. Поэтому в рассматриваемом случае возникает вопрос по обоснованному выбору величины $f$ для стоячей поперечной ЭМВ в металлическом проводнике. В дальнейшем будем исходить из того предположения, что в металлическом проводнике частота колебаний $f$ стоячей поперечной ЭМВ в обобщенном виде может изменяться в интервале $\left[0, f_{0}\right]$ (на наружной поверхности проводника при $r=r_{0}$ частота колебаний ЭМП равна $f_{0}$, а вдали от нее при $r \rightarrow 0$ для массивного тела круглого проводника $f \rightarrow 0$ ) и удовлетворять для $0 \leq t \leq f_{0}^{-1} \quad$ следующей функции:

$$
f=f_{0} \times\left|\sin \left(2 \pi f_{0} t\right)\right| .
$$

Исходя из (11), для среднего квадратичного значения частоты колебаний $f$ стоячей поперечной ЭМВ в металле проводника за период колебаний $T_{0}=1 / f_{0}$ тока проводимости $i_{0}(t)$ в нем имеем:

$$
f_{0 m}=\left[f_{0} \int_{0}^{f_{0}^{-1}} f^{2} d t\right]^{1 / 2}=f_{0} /(2)^{1 / 2} .
$$

Используя (12) для определения усредненной скорости $v_{m r}$ распространения плоскомеридианной стоячей поперечной ЭМВ в рассматриваемом проводнике с током проводимости $i_{0}(t)$, нам необходимо оценить погрешность, вносимую в расчет скорости $v_{m r}$ ЭМВ указанного вида в его материале принятым электродинамическим приближением. При этом будем исходить из того положения, что частота изменения $f$ в металле (сплаве) проводника гармонических колебаний напряженностей электрического и магнитного полей в стоячей поперечной ЭМВ в первом приближении соответствует, согласно (12), усредненной частоте колебаний $f_{0 m}=f_{0} /(2)^{1 / 2}$, где $f_{0}-$ частота колебаний тока проводимости $i_{0}(t)$ в проводнике. Из соотношения $\lambda_{r} \approx v_{m r} / f_{0 m}$ с учетом (12) видно, что при таком определении величины $v_{m r} \approx \lambda_{r} f_{0} /(2)^{1 / 2}$ основную погрешность в ее расчет будет вносить неопределенность (точность) нахождения частоты $f_{0}$ колебаний тока $i_{0}(t)$, протекающего по проводнику. В силовых электрических цепях промышленного электроснабжения изменения частоты $f_{0}=50$ Гц переменного тока (напряжения) не превышают 1\% [12]. В электрических цепях высоковольтной импульсной техники (ВИТ), использующих емкостные накопители энергии, неопределенность частоты колебаний $f_{0}$ разрядного импульсного тока $i_{0}(t)$ в токоведущих частях электроустановок составляет не более погрешности ее измерения с помощью запоминающих цифровых осциллографов (максимум 3\%) [13].

Учитывая вышеизложенное, при расчетной оценке усредненной скорости $v_{m r}$ распространения стоячих поперечных ЭМВ в металле (сплаве) проводника после подстановки величины $\lambda_{r}$ из (10) в соотношение вида $\lambda_{r} \approx(2)^{1 / 2} v_{m r} / f_{0}$ можно воспользоваться следующей приближенной формулой:

$$
v_{m r} \approx\left[2 \pi f_{0} /\left(\mu_{0} \gamma_{0}\right)\right]^{1 / 2} .
$$

Из (13) для медного проводника $\left(\gamma_{0}=5,81 \times 10^{7} \mathrm{Cm} / \mathrm{M}[7]\right)$ при $f_{0}=50$ кГц (электрофизический случай, характерный для сильноточных разрядных цепей ВИТ [13]) следует, что $v_{m r} \approx 65,6 \mathrm{~m} / \mathrm{c}$. Видно, что усредненная скорость $v_{m}$ распространения стоячих поперечных ЭМВ в немагнитной металлической среде исследуемого проводника на много порядков оказывается меньше средней скорости распространения того же переменного ЭМП в воздушной среде $\left(v_{0}=1 /\left(\varepsilon_{0} \mu_{0}\right)^{1 / 2} \approx 3 \times 10^{8} \mathrm{M} / \mathrm{c}, \quad\right.$ где $\varepsilon_{0}=8,854 \times 10^{-12} \Phi / \mathrm{M}$ - электрическая постоянная [7]), окружающей рассматриваемый металлический проводник.

\section{РАСЧЕТНАЯ ОЦЕНКА УСРЕДНЕННОЙ СКОРОСТИ РАСПРОСТРАНЕНИЯ СТОЯЧИХ ЭЛЕКТРОННЫХ ВОЛН ДЕ БРОЙЛЯ В МАТЕРИАЛЕ ПРОВОДНИКА}

Квантованные стоячие продольные электронные волны де Бройля в металле (сплаве) рассматриваемого немассивного цилиндрического проводника с аксиальным током проводимости $i_{0}(t)$ характеризуются усредненным значением своей длины $\lambda_{m z}$, имеющим следующий вид [13]:

$$
\lambda_{m z} \approx 2 l_{0} \ln n_{m} / n_{m} .
$$

Согласно (14), для исследуемого круглого стального провода в случае, когда $r_{0} \approx 0,8$ мм, 
$l_{0} \approx 320$ мм и $n_{m}=32$, усредненная длина $\lambda_{m z}$ продольной электронной волны де Бройля в его проводящем материале численно составляет около 69,3 мм. Следует указать на то, что правомерность расчетного соотношения (14) была подтверждена экспериментальными данными, приведенными в [5, 13], применительно к указанному электрофизическому случаю при опытном изучении в области ВИТ волн де Бройля.

Так как продольные дебройлевские электронные волны в исследуемом проводнике обусловлены продольным дрейфом коллективизированных свободных электронов его металла (сплава), то при своей усредненной длине $\lambda_{m z}$, рассчитываемой по (14), они (волны де Бройля) будут иметь усредненную квантованную скорость $v_{n z}=v_{m z}$ распространения в материале данного проводника. Эта усредненная скорость $v_{m z}$ продольного дрейфа свободных электронов проводника будет удовлетворять фундаментальному соотношению квантовой физики, имеющему для продольной электронной волны де Бройля в металле (сплаве) исследуемого проводника с током проводимости $i_{0}(t)$ применительно к рассматриваемому случаю определения ее усредненных параметров следующий аналитический вид [6]:

$$
v_{m z}=h /\left(m_{e} \lambda_{m z}\right),
$$

где $h=6,626 \times 10^{-34}$ Дж·с - постоянная Планка [7]; $m_{e}=9,109 \times 10^{-31}$ кг - масса покоя электрона [7].

После подстановки (14) в (15) для усредненной скорости $v_{m z}$ распространения стоячих продольных электронных волн де Бройля в рассматриваемом металлическом проводнике имеем:

$$
v_{m z} \approx n_{m} h /\left(2 m_{e} l_{0} \ln n_{m}\right) .
$$

Из (16) для реального случая, описанного в $[4,5]$ при опытном исследовании в разрядной цепи мощного высоковольтного генератора импульсных токов $i_{0}(t)$ продольных ВЭП в круглом стальном проводе $\left(r_{0} \approx 0,8\right.$ мм; $l_{0} \approx 320 \mathrm{MM} ; n_{m}=32 ; \quad I_{0 m} \approx 745 \mathrm{~A}$; $\delta_{0 m} \approx 0,37 \mathrm{\kappa A} / \mathrm{MM}^{2}$ ), получаем, что усредненная скорость $v_{m z}$ распространения продольных электронных волн де Бройля в этом стальном проводе с апериодическим импульсом тока временной формы 9 мс/160 мс будет составлять около $10,5 \mathrm{~mm} / \mathrm{c}$.

Из анализа полученных результатов для усредненных скоростей $v_{n r}=v_{m r}$ и $v_{n z}=v_{m z}$ распространения в однородном металле (сплаве) рассматриваемого проводника соответственно квантованных стоячих поперечных ЭМВ и стоячих продольных электронных волн де
Бройля следует неравенство вида $v_{m r}>v_{m z}$. Кроме того, указанные усредненные скорости $v_{m r}$ и $v_{m z}$ оказываются существенно меньшими средней скорости $v_{0}$ распространения бегущей поперечной ЭМВ в воздушной среде, окружающей рассматриваемый проводник $\left(v_{0} \approx 3 \times 10^{8} \mathrm{M} / \mathrm{c}\right.$ [7]). Эти электродинамические данные могут обоснованно указывать на то, что волновые электромагнитные процессы значительно быстрее протекают и устанавливаются в воздушном диэлектрике вне проводящего материала исследуемого проводника с переменным током проводимости $i_{0}(t)$ различных АВП по сравнению с их весьма медленным протеканием и установлением в однородном металле (сплаве) рассматриваемого проводника.

\section{ЗАКЛЮЧЕНИЕ}

1. На основе приближенного определения продольного волнового распределения дрейфующих свободных электронов в изотропном металле (сплаве) круглого цилиндрического проводника конечных размеров (радиусом $r_{0}$ и длиной $l_{0}>r_{0}$ ) с электрическим переменным аксиальным током проводимости $i_{0}(t)$ различных АВП получены соотношения (13) и (16) для расчетной оценки усредненных скоростей распространения $v_{m r}$ и $v_{m z}$ соответственно для квантованных стоячих поперечных ЭМВ и квантованных стоячих продольных электронных волн де Бройля в рассматриваемом проводнике.

2. Показано, что возникающие в металле (сплаве) исследуемого немассивного проводника конечных размеров квантованные стоячие поперечные ЭМВ существенно отличаются от обычных поперечных ЭМВ, распространяющихся в массивных линейных проводящих средах неограниченных геометрических размеров. При этом принципиальной особенностью для стоячих поперечных ЭМВ в рассматриваемом металлическом проводнике с током проводимости $i_{0}(t)$ является то, что их напряженность $E_{n z}(z, r, t)$ продольного электрического поля опережает по фазе в ЭМВ соответствующую напряженность $H_{n v}(z, r, t)$ азимутального магнитного поля на угол, численно равный $\pi / 2$.

3. Установлено, что квантованные стоячие поперечные ЭМВ с указанными компонентами напряженностей переменного ЭМП не переносят по металлу (сплаву) исследуемого проводника с электрическим аксиальным током проводимости $i_{0}(t)$ различных АВП потоков электромагнитной энергии. В этих квантованных стоячих поперечных ЭМВ энергия их продольного электрического поля с напряженностью $E_{n z}(z, r, t)$ 
будет без потерь периодически переходить в энергию азимутального магнитного поля с напряженностью $H_{n v}(z, r, t)$ и наоборот.

4. Полученные результаты позволяют сделать определенный шаг в дальнейшем развитии теории волновых электромагнитных процессов, протекающих в металлическом проводнике конечных размеров с электрическим током проводимости $i_{0}(t)$ произвольных АВП. Установлено, что данные электромагнитные процессы значительно быстрее протекают в диэлектрике вне проводящего материала проводника по сравнению с их достаточно медленным установлением в металле (сплаве) проводника.

\section{КОНФЛИКТ ИНТЕРЕСОВ}

Автор заявляет, что у него нет конфликта интересов.

\section{ЛИТЕРАТУРА}

1. Baranov, M.I., Wave distribution of free electrons in a current-bearing conductor, Russ. Electr. Eng., 2005, vol. 76, no. 7, p. 30.

2. Баранов, М.И., Новые физические подходы и механизмы при изучении процессов формирования и распределения электрического тока проводимости в проводнике. Технічна електродинаміка, 2007, № 1, с. 13.

3. Баранов, М.И., Квантово-волновая природа электрического тока в металлическом проводнике и еe некоторые электрофизические макропроявления, Електротехніка $i$ електромеханіка, 2014, № 4, c. 25 . doi: 10.20998/2074272X.2014.4.05.

4. Баранов, М.И., Основные характеристики волнового распределения свободных электронов в тонком металлическом проводнике с импульсным током большой плотности, Электричество, 2015, № 10, c. 20.

5. Baranov, M.I., Rudakov, S.V., Calculationexperimental method of research in a metallic conductor with the pulse current of electronic wavepackages and de Broglie electronic half-waves, Electrical engineering \& electromechanics, 2016, no. 6, p. 45. doi: 10.20998/2074-272X.2016.6.08.

6. Кузьмичев, В.Е., Законы и формуль физики. Отв. ред. В.К. Тартаковский. Киев: Наукова думка, 1989. $864 \mathrm{c}$.

7. Кухлинг, Х., Справочник по физике. Пер. с нем. под ред. Е.М. Лейкина, М.: Мир, 1982. 520 с.
8. Никольский, В.В., Никольская, Т.И., Электродинамика и распространение радиоволн. Учебное пособие для вузов, М.: Наука, 1989. 544 с.

9. Демирчян, К.С., Нейман, Л.Р., Коровкин, Н.В., Чечурин, В.Л., Теоретические основы электротехники. Учебник для вузов в 3 томах. Том 3. Санкт-Петербург: Питер, 2004. 377 с.

10. Кнопфель, Г., Сверхсильные импульсные магнитные поля. Пер. с англ. Ф.А. Николаева, Ю.П. Свириденко, М.: Мир, 1972. 391 с.

11. Нейман, Л.Р., Демирчян, К.С., Теоретические основы электротехники. Учебник для вузов в 2 томах. Том 2. Л.: Энергоиздат, 1981.416 с.

12. Электротехнический справочник. Производство и распределение электрической энергии. Том 3. Книга 1. Под общей ред. И.Н. Орлова. М.: Энергоатомиздат, 1988. $880 \mathrm{c}$.

13. Баранов, М.И., Избранные вопросы электрофизики. Монография в 4 томах. Том 2. Книга 1. Теория электрофизических эффектов и задач. Харьков: НТУ “ХПІ”, 2009. 384 с.

\section{Summary}

The paper demonstrates the results of approximate calculations on the establishment of basic features of the propagation of standing transversal electromagnetic waves (EMWs) and standing longitudinal de Broglie electronic waves in a homogeneous not massive nonmagnetic metallic conductor of finite dimensions (the radius $r_{0}$ and the length $l_{0}>>r_{0}$ ) with the alternating axial-flow current of conductivity of $i_{0}(t)$ of different peak-temporal parameters. The correlation for the rated estimation of the average velocity of propagation of the standing transversal EMWs and standing longitudinal de Broglie electronic waves in a metal (alloy) of the indicated conductor is presented. It is shown that quantized standing transversal EMWs arising in a metallic conductor of finite dimensions substantially differ from ordinary transversal EMWs, propagated in the conducting environments of unlimited dimensions. An important feature of the standing transversal EMWs in the examined conductor is the fact that their tension of an axial-flow electric-field advances by a phase their tension of an azimuthal magnetic-field on the corner of $\pi / 2$. It was established that in the standing transversal EMWs of the used conductor the energy of their electric field only passes into the energy of their magnetic field and vice versa. Therefore the standing transversal EMWs do not transfer the flows of the electromagnetic energy on the surface of the studied conductor.

Keywords: metallic conductor, alternating conduction current, standing transversal electromagnetic waves, standing longitudinal de Broglie electronic waves, velocity of waves propagation 